\title{
Patient considerations and clinical impact of cholesteryl ester transfer protein inhibitors in the management of dyslipidemia: focus on anacetrapib
}

\author{
This article was published in the following Dove Press journal: \\ Vascular Health and Risk Management \\ 22 August 2012 \\ Number of times this article has been viewed
}

\section{Marta A Miyares \\ Kyle Davis}

Pharmacy Department, Jackson Memorial Hospital, Miami, FL, USA
Correspondence: Marta A Miyares Pharmacy Department, Jackson Memorial Hospital, I6II NW I2 Avenue, East Tower B069, Miami,

FL 33।36- 1096, USA

Tel +I 3055857454

Emailmmiyares@jhsmiami.org
Abstract: Cardiovascular disease (CVD) is responsible for significant morbidity and mortality within the United States and worldwide. Although targeting low-density lipoprotein cholesterol (LDL-C) in the prevention of CVD has been shown to be effective, evidence exists to indicate that significant cardiovascular (CV) risk remains in patients receiving 3-hydroxy-3-methylglutarylcoenzyme A reductase inhibitors (statins) - a risk that may be correlated with low levels of high-density lipoprotein cholesterol (HDL-C). Among the various tactics under investigation to increase HDL-C, inhibition of cholesteryl ester transfer protein (CETP) appears the most adept to raise these levels. Although torcetrapib, a CETP inhibitor, demonstrated significant beneficial changes in HDL-C and LDL-C after 12 months of therapy when coadministered with atorvastatin, patients in the torcetrapib arm experienced a rise in mortality, including increased risk of death from $\mathrm{CV}$ and non-CV causes as well as a significant rise in major $\mathrm{CV}$ events. Later studies established that the adverse effects of torcetrapib were produced from molecule-specific off-target effects and not to the mechanism of CETP inhibition. These untoward outcomes have not been detected with anacetrapib, the third of the CETP inhibitors to enter Phase III trials. Furthermore, treatment with anacetrapib revealed both a statistically significant decrease in LDL-C and increase in HDL-C over placebo. While the place in therapy of niacin and fibrates to reduce $\mathrm{CV}$ events is currently in question secondary to the Atherothrombosis Intervention in Metabolic Syndrome with Low HDL Cholesterol/High Triglyceride and Impact on Global Health Outcomes and the Action to Control CV Risk in Diabetes trials, the ongoing large-scale, randomized-placebo, controlled-outcomes study with anacetrapib coadministered with statin treatment will not only test the hypothesis if CETP inhibition lowers residual CV risk but will also provide insight as to which patient subgroups might benefit the most from anacetrapib despite aggressive therapy with statins.

Keywords: anacetrapib, cardiovascular disease, cholesteryl ester transfer protein, cholesteryl ester transfer protein inhibitor, dyslipidemia

Cardiovascular disease (CVD) is responsible for significant morbidity and mortality within the United States (US) and worldwide. The prevalence of CVD in the US is projected to be $37.8 \%$ by 2015 - an estimate which will only increase in subsequent years. ${ }^{1}$

Current guidelines for the prevention of coronary heart disease (CHD) identify low-density lipoprotein cholesterol (LDL-C) as the primary target for lipid-lowering therapy. ${ }^{2,3}$ Numerous randomized controlled clinical trials have solidified 3-hydroxy3-methylglutaryl-coenzyme A (HMG-CoA) reductase inhibitors (statins) as the cornerstone of LDL-C-lowering therapy. More importantly, in addition to lowering 
surrogate levels of LDL-C, the use of statins in the primary and secondary prevention of CVD have been linked to significant reductions in cardiovascular $(\mathrm{CV})$ outcomes and all-cause mortality. ${ }^{4-6}$ These reductions are believed to stem from the pleiotropic effects of statins in addition to their LDL-C-lowering ability. ${ }^{\text {? }}$

Although targeting LDL-C in the prevention of CVD has been shown to be effective, evidence exists to indicate that significant $\mathrm{CV}$ risk remains in patients receiving statin-based therapy - a risk that may be correlated with subsequently low levels of high-density lipoprotein cholesterol (HDL-C). In a post hoc analysis of the Treating to New Targets study in which randomized patients received atorvastatin $10 \mathrm{mg}$ daily or atorvastatin $80 \mathrm{mg}$ daily, the frequency of major $\mathrm{CV}$ events increased with decreasing levels of HDL-C in both treatment arms. ${ }^{8}$ This relationship was seen even among patients obtaining LDL-C levels less than $70 \mathrm{mg} / \mathrm{dL}$. This inverse relationship between HDL-C and the risk of CVD has long been established through several epidemiological studies. ${ }^{9-12}$ The landmark Framingham Study concluded that HDL-C was the most significant lipid risk factor for CHD. ${ }^{9}$ One evaluation of four epidemiologic studies put forward that for each $1 \mathrm{mg} / \mathrm{dL}$ increase in HDL-C, there was a decrease in CHD of $1.9 \%-2.9 \% .{ }^{13}$ As a result of this epidemiological evidence, targeting HDL-C to further reduce $\mathrm{CV}$ risk provides an appealing alternative to LDL-C-lowering therapy.

\section{Cholesteryl ester transfer protein inhibitors}

Among the various tactics under investigation to increase HDL-C, inhibition of cholesteryl ester transfer protein (CETP) appears the most adept to raise such levels. ${ }^{14,15}$ Initially, torcetrapib (CP-529414; Pfizer, La Jolla, CA), a CETP inhibitor, demonstrated promising results. In a Phase I trial conducted in healthy volunteers, torcetrapib at doses of $120 \mathrm{mg}$ once daily and twice daily for 14 days increased HDL-C by $73 \%$ and $91 \%$, respectively, and decreased LDL-C by $21 \%$ and $42 \%$, respectively, with no evident adverse effects. ${ }^{16}$ When torcetrapib $120 \mathrm{mg}$ daily was administered with atorvastatin $20 \mathrm{mg}$ daily, an LDL-C reduction of $17 \%(P=0.02)$ beyond that achieved with atorvastatin alone and an HDL-C increase of $61 \%$ ( $P<0.001)$ occurred after 4 weeks. ${ }^{17}$ Eventually, early trials brought torcetrapib under scrutiny when results demonstrated an elevation in systolic blood pressure (SBP) and diastolic blood pressure (DBP) of 1.3 to 2.2 and 0.9 to $1.1 \mathrm{mmHg}$ at doses of 60 or $90 \mathrm{mg}$ daily, respectively. Consequently, future trials with torcetrapib were restricted to utilize a dose of $60 \mathrm{mg}$ daily. ${ }^{18,19}$
In the fourth quarter of 2006, all the torcetrapib trials were suspended due to the results of the Investigation of Lipid Level Management to Understand Its Impact in Atherosclerotic Events (ILLUMINATE) trial, which enrolled 15,067 high-risk CV patients. The participants were randomized to receive either atorvastatin 10 to $80 \mathrm{mg}$ daily and placebo or atorvastatin and torcetrapib $60 \mathrm{mg}$ daily. Despite a $72.1 \%$ increase in HDL-C and a $24.9 \%$ decrease in LDL-C after 12 months of therapy with the combination regimen, patients in the torcetrapib arm experienced a rise in mortality, including increased risk of death from both $\mathrm{CV}$ and non-CV causes as well as a significant rise in major $\mathrm{CV}$ events of $25 \%(95 \%$ confidence interval $[\mathrm{CI}]: 1.09-1.44 ; P=0.001) .{ }^{20}$ These results were confirmed by simultaneous trials: Investigation of Lipid Level Management Using Coronary Atherosclerosis by CETP Inhibition and HDL Elevation (ILLUSTRATE), Rating Atherosclerosis Disease Change with a New CETP Inhibitor (RADIANCE)-1 and RADIANCE-2. ${ }^{21-23}$

Later studies established that the adverse effects of torcetrapib were produced from molecule-specific off-target effects and not to the mechanism of CETP inhibition. ${ }^{24-26}$ Regardless of the 60-mg dose cap per day in ILLUMINATE, ILLUSTRATE, RADIANCE-1, and RADIANCE-2, the mean SBP elevations were 5.4, 4.6, 2.8, and $5.4 \mathrm{mmHg}$, respectively. ${ }^{20-23}$ Further analyses of ILLUSTRATE, RADIANCE-1, and RADIANCE-2 pointed to a mineralcorticoid effect accompanied by an elevation in serum sodium and decreased serum potassium in patients who received torcetrapib. Forrest et al demonstrated that torcetrapib increased blood pressure through a CETP-independent pathway in mice (both with and without a CETP transgene), rats, dogs, and rhesus monkeys. ${ }^{26}$ These untoward outcomes have not been detected with the other two CETP inhibitors, anacetrapib (MK-0859; Merck, Whitehouse Station, NJ) or dalcetrapib (JTT-705; Roche, Nutley, NJ), both of which entered Phase III trials. ${ }^{27}$

Dalcetrapib was halted in May 2012 due to lack of efficacy in the Phase III dAL-OUTCOMES trial, a study in stable CHD patients with recent acute coronary syndrome. ${ }^{28}$ In comparison to the other CETP inhibitors, anacetrapib and torcetrapib, dalcetrapib was a significantly less potent inhibitor of CETP. ${ }^{29}$ Evacetrapib (LY2484595; Eli Lilly, Indianapolis, IN), DRL-17822 (Dr Reddy's Laboratories, Hyderabad, India), and JTT-302 (Japan Tobacco, Tokyo, Japan) are currently undergoing Phase II investigation, while AT-103 (AFFiRiS AG, Vienna, Austria), a vaccine against CETP, and TA-8995 (Mitsubishi Tanabe, Osaka, Japan) are in early stage development. Anacetrapib, the third of the CETP 
inhibitors to commence Phase III trials, will be discussed in detail in this manuscript.

\section{The role of CETP in cholesterol metabolism}

Cholesterol is maintained by means of two homeostatic processes that lead cholesterol away from and back to the liver. Lipids secreted from hepatocytes in the form of very low-density lipoprotein cholesterol (VLDL-C), intermediatedensity lipoprotein cholesterol, and LDL-C particles are taken up by the peripheral tissues via the LDL receptor. Alternatively, excess cholesterol in the periphery is removed and transported back to the liver by HDL-C via scavenger receptors for recycling and excretion from the body. This mechanism is most commonly termed reverse cholesterol transport (RCT) (Figure 1). ${ }^{30,31}$ The role HDL-C plays in the removal of cholesterol from the body by means of RCT is essential in maintaining cholesterol equilibrium and is believed to subsequently give HDL-C its atheroprotective properties. Further contributing to the beneficial effects of HDL-C mediated RCT are the anti-inflammatory, antioxidative, antiapoptotic, antithrombotic, vasodilatory, and anti-infectious properties. ${ }^{32}$
Within the RCT pathway exists CETP, a hepatically derived hydrophobic glycoprotein secreted from the liver that binds to HDL-C. CETP mediates the equimolar transfer of cholesteryl ester (CE) from HDL to apolipoprotein (apo) B lipoproteins (chylomicra, VLDL-C, and LDL-C) and the equimolar transfer of triglycerides (TGs) from VLDL-C and LDL-C to HDL-C. ${ }^{33}$ The involvement of CETP in the RCT pathway is believed to result in both antiatherogenic and atherogenic activity. CETP-mediated transfer of CE accounts for the return of cholesterol from the peripheral cells to the liver via LDL receptors. Cholesterol can then be secreted into bile and eliminated from the body, leading to potentially antiatherogenic results. Conversely, when LDL receptors are unable to function adequately, CETP leads to the accumulation of LDL-C in the plasma. ${ }^{34}$ The exchange of CE from HDL-C to apo B lipoproteins may also prevent efflux of cholesterol from peripheral cells and diminish circulating levels of HDL-C, which can reduce return of cholesterol from the arterial walls back to the liver. ${ }^{35} \mathrm{In}$ addition, CETP activity causes HDL-C and LDL-C to become TG-heavy, giving way to small-dense HDL-C and LDL-C. The small-dense LDL-C has an increased susceptibility to oxidative modification by

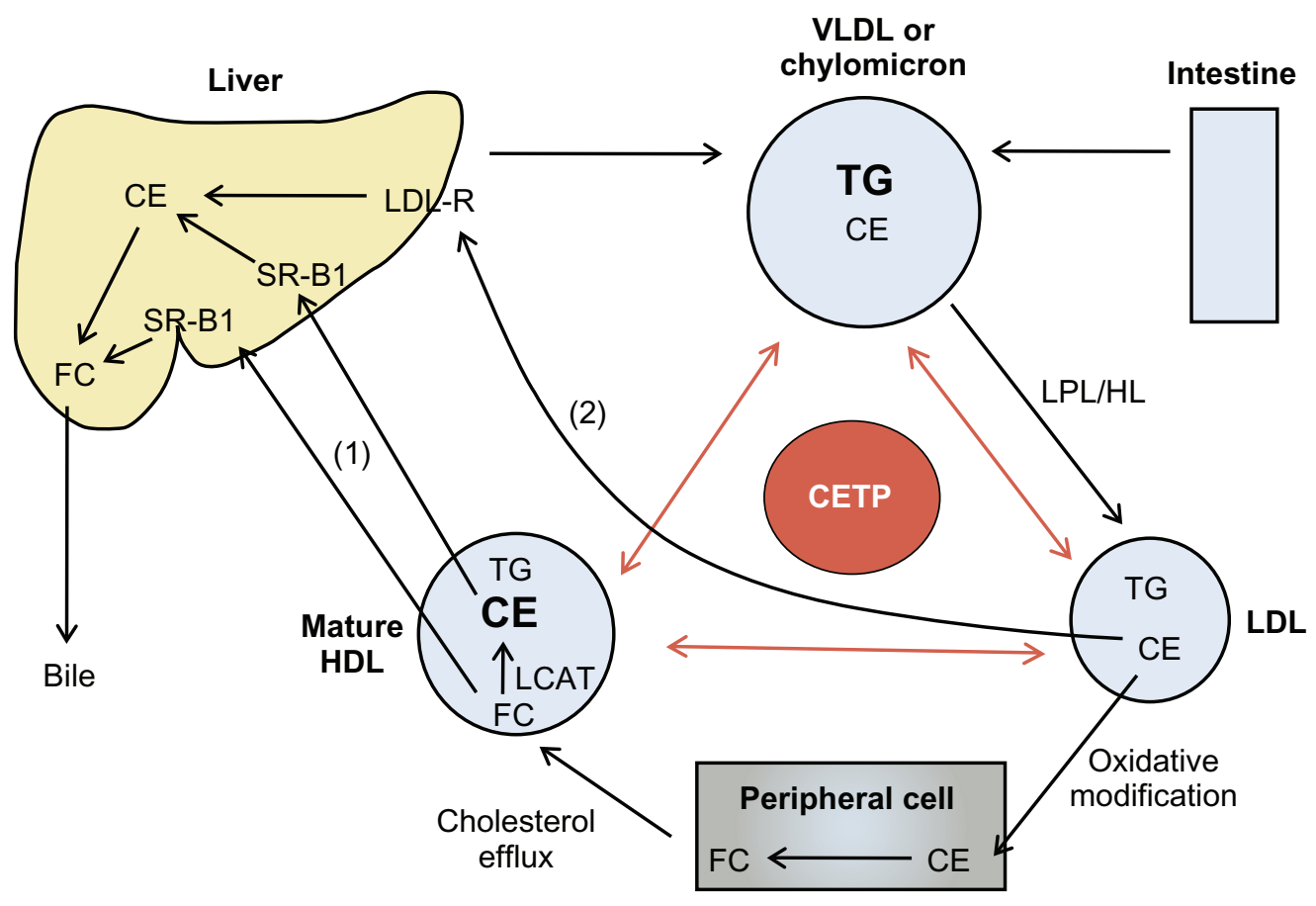

Figure I The function of CETP in RCT.

Notes: CETP mediates the equimolar transfer of CE and TG between atherogenic VLDL, LDL, and anti-atherogenic HDL. The majority of CE originates in HDL-C via a reaction carried out by LCAT, whereas the bulk of TG stems from the liver and intestine as VLDL or chylomicrons, respectively. LPL and HL hydrolyze a portion of TG from VLDL, which converts VLDL to LDL. Cells within the liver and peripheral tissue that express the LDL receptor take up excess LDL. In addition, macrophages also take up LDL and are converted to foam cells. Esterified and free (unesterified) cholesterol (FC) is then taken up by HDL via the SR-BI (Pathway I) and by LDL via the LDL-R (pathway 2). Reprinted from the Journal of the American College of Cardiology. Barter PJ, Kastelein JJ. Targeting cholesteryl ester transfer protein for the prevention and management of cardiovascular disease. J Am Coll Cardiol. 2006;47(3):492-499. Copyright ${ }^{\odot} 2006$, with permission from Elsevier. ${ }^{31}$

Abbreviations: CE, cholesteryl ester; CETP, cholesteryl ester transfer protein; FC, free cholesterol; HDL, high-density lipoprotein; HDL-C, high-density lipoprotein cholesterol; HL, hepatic lipase; LCAT, lecithin cholesterol acyltransferase; LDL, low-density lipoprotein; LDL-R, low-density lipoprotein receptor; LPL, lipoprotein lipase; RCT, reverse cholesterol transport; SR-BI, scavenger receptor-BI; TG, triglyceride; VLDL, very low-density lipoprotein. 
TG lipase, which promotes uptake within the arterial wall by macrophage scavenger receptors. ${ }^{36}$ Predominance of this smaller less buoyant LDL-C has been associated with up to a threefold increased risk of developing CAD. ${ }^{37}$ Considering the potentially atherogenic effects of CETP, inhibition of this protein and the ability to increase antiatherogenic HDL-C has generated great interest in the prevention and treatment of CAD and atherosclerosis.

The correlation between CETP deficiencies and CV risk remains unclear at this point. Initial studies indicated that deficiencies in CETP were associated with an increased risk of CAD. ${ }^{38,39}$ However, the Women's Genome Health Study revealed opposing results, suggesting cardioprotective benefits of CETP inactivity through an HDL-C-mediated pathway. ${ }^{40}$ Finally, results of the Honolulu Heart Study revealed that men with CETP mutations had the lowest risk of CHD; however, these results were not statistically significant. ${ }^{41}$ Therefore, the outcomes of these trials and the potential CV benefit of CETP modulation merit further research in this field.

Anacetrapib is a once daily, orally administered, CETP inhibitor that is currently undergoing Phase III clinical trials. Similar to torcetrapib, anacetrapib exhibits its effect by forming a reversible bond between CETP and HDL-C. Formation of this bond results in the inhibition of CETPmediated CE and TG transfer between HDL-C and apo B lipoproteins, consequently increasing circulating antiatherogenic HDL-C. ${ }^{27}$ In comparison to the other CETP inhibitors, torcetrapib and dalcetrapib, anacetrapib shares a similar potency with torcetrapib and is a significantly more potent inhibitor of CETP than dalcetrapib. ${ }^{29}$

\section{Pharmacokinetics, drug interactions, and pharmacodynamics}

The pharmacokinetics of anacetrapib have been evaluated in healthy humans, dyslipidemic subjects, and animal models. ${ }^{25,42-45}$ Following a 1-hour delay, anacetrapib is promptly absorbed with peak concentrations arising approximately 4 hours after administration. ${ }^{25,43-45}$ Although anacetrapib is highly plasma protein-bound, the binding is reversible. After a 150-mg dose was administered in dyslipidemic patients, the mean maximum concentration $\left(\mathrm{C}_{\max }\right)$ of anacetrapib in plasma was $1861 \mathrm{nM}$ on day 1 and $1960 \mathrm{nM}$ on day 28 , depicting a similar concentration after once-daily multiple dosing compared to a single dose upon initiation. ${ }^{25}$ While the pharmacokinetic profile of anacetrapib is not affected by age, sex, or obesity, ${ }^{43}$ food significantly enhances the absorption. A low-fat meal increased area under the curve
$(\mathrm{AUC})_{0-\infty}$ up to two times and $\mathrm{C}_{\max }$ up to three times, while a high-fat meal increased $\mathrm{AUC}_{0-\infty}$ up to six times and $\mathrm{C}_{\max }$ up to nine times versus the fasted state. ${ }^{43}$ Therefore, anacetrapib is recommended to be administered with meals. ${ }^{25,43,44}$ Steady state is reached after 7 days. ${ }^{25,44}$ The three minor radioactive metabolites formed through cytochrome P450 (CYP)3A4catalyzed oxidation are excreted by the biliary-fecal route. ${ }^{45}$ Anacetrapib has a biphasic elimination profile. It has a long terminal half-life and it exhibits an effective half-life of approximately 18 hours. ${ }^{25,44}$ A study by Dansky et al concluded that even after cessation of anacetrapib 8 weeks prior, the continued presence of drug levels coupled with persistent decreases in LDL-C and residual elevations in HDL-C suggested that anacetrapib has a terminal half-life of approximately 3 to 4 weeks. ${ }^{46}$

When Krishna et al assessed the CYP3A drug interaction potential of anacetrapib in healthy volunteers, the medication did not influence the activity of this enzyme. ${ }^{47}$ The study did provide evidence that anacetrapib is a moderately sensitive substrate of CYP3A as the plasma samples revealed that anacetrapib activity was elevated by ketoconazole, a potent CYP3A4 inhibitor. Additionally, the pharmacokinetics of drugs that are substrates for CYP pathways are not modified by anacetrapib. When midazolam was used as a probesensitive CYP3A substrate, anacetrapib treatment did not affect the activity of this enzyme, as depicted by a lack of variance in the plasma midazolam concentration. Similarly, a study evaluating the pharmacokinetic effects of simvastatin, also a sensitive CYP3A substrate, administered as monotherapy or in combination with anacetrapib, revealed no difference. ${ }^{48}$ Subsequent to these trials, studies have been performed with digoxin and warfarin. ${ }^{49,50}$ Digoxin is a substrate of the P-gylcoprotein (Pgp)-mediated transport pathway. Metabolism of digoxin does not rely on nor does it affect CYP metabolism. Multiple dose administration of anacetrapib $100 \mathrm{mg}$ did not affect single-dose pharmacokinetics of digoxin or warfarin in healthy treatment groups. Therefore, no dosage adjustment is required for warfarin or digoxin when used concomitantly with anacetrapib.

Pharmacodynamic studies to analyze the serum CETP inhibitory potential of anacetrapib have been completed. ${ }^{43,44}$ A study by Krishna et al revealed that while anacetrapib exerted a serum CETP maximum inhibition of nearly $90 \%$ at 4 hours on the first day, the inhibitory potential decreased $5 \%$ to $10 \%$ at the same time point after 14 days. On day 1 , the trough inhibitory potential was approximately $80 \%$, and after 14 days of repeated doses, the inhibitory potential was diminished about $20 \%{ }^{44}$ An elucidation of this 
occurrence may be due to the threefold increase in CETP concentration observed throughout the study. Although a precise explanation as to the CETP mass increase is not confirmed, Clark et al credited this increase to the strong adherence of CETP for HDL-C with development of a nonproductive complex ${ }^{51}$ Clark et al further noted that the CETP mass increase should not interfere with HDL-C elevation as the emergence of CETP concentration is not significant compared to the amount of HDL particles. This theory was validated when anacetrapib was dosed for 4 weeks and no associated loss of efficacy was found. ${ }^{25}$

\section{Clinical trials}

The effects of anacetrapib on surrogate markers of CVD when administered alone or in combination with atorvastatin have been examined by Bloomfield et al..$^{52}$ The study enrolled 589 adult patients with primary hypercholesterolemia or mixed hyperlipidemia who had LDL-C between 100 to $190 \mathrm{mg} / \mathrm{dL}, 100$ to $160 \mathrm{mg} / \mathrm{dL}$ if categorized as moderaterisk patients, or 100 to $130 \mathrm{mg} / \mathrm{dL}$ if diabetic. The majority (53.8\%) of patients had low HDL-C (mean, $50.5 \mathrm{mg} / \mathrm{dL}$ ) and a median LDL-C of $141.1 \mathrm{mg} / \mathrm{dL}$ at baseline. Patients were randomized to one of ten groups: placebo, atorvastatin $20 \mathrm{mg}$ alone, anacetrapib 10, 40, 150, or $300 \mathrm{mg}$ once daily as monotherapy, or atorvastatin $20 \mathrm{mg}$ in combination with anacetrapib at the aforementioned dosage strengths for 8 weeks. Each of the groups included an equal number of patients with TG greater than $150 \mathrm{mg} / \mathrm{dL}$.

The efficacy endpoints in the study were the percent change from baseline within the different treatment groups in LDL-C (primary), HDL-C, non-HDL-C, total cholesterol, TG, and apo B, apo A-I, and apo E (secondary). Both HDL-C and LDL-C were statistically significantly increased and decreased, respectively, when anacetrapib was administered alone or in combination with atorvastatin (Figures 2 and 3). Additionally, although similar to the percentage change with anacetrapib monotherapy, there was a statistically significant increase in HDL-C with combination treatment versus atorvastatin monotherapy (Figure 3). There was no further benefit in terms of lipid alteration when the anacetrapib dose was increased from 150 to $300 \mathrm{mg}$ (Figures 2 and 3). Total cholesterol and TG were not affected by anacetrapib monotherapy or with concomitant administration with atorvastatin. Apo E and apo A-I were increased and lipoprotein (a) levels were decreased with elevating doses of anacetrapib. The C-reactive protein reduction of $30.9 \%$ with atorvastatin monotherapy was attenuated with combination treatment. All of the treatment arms tolerated anacetrapib
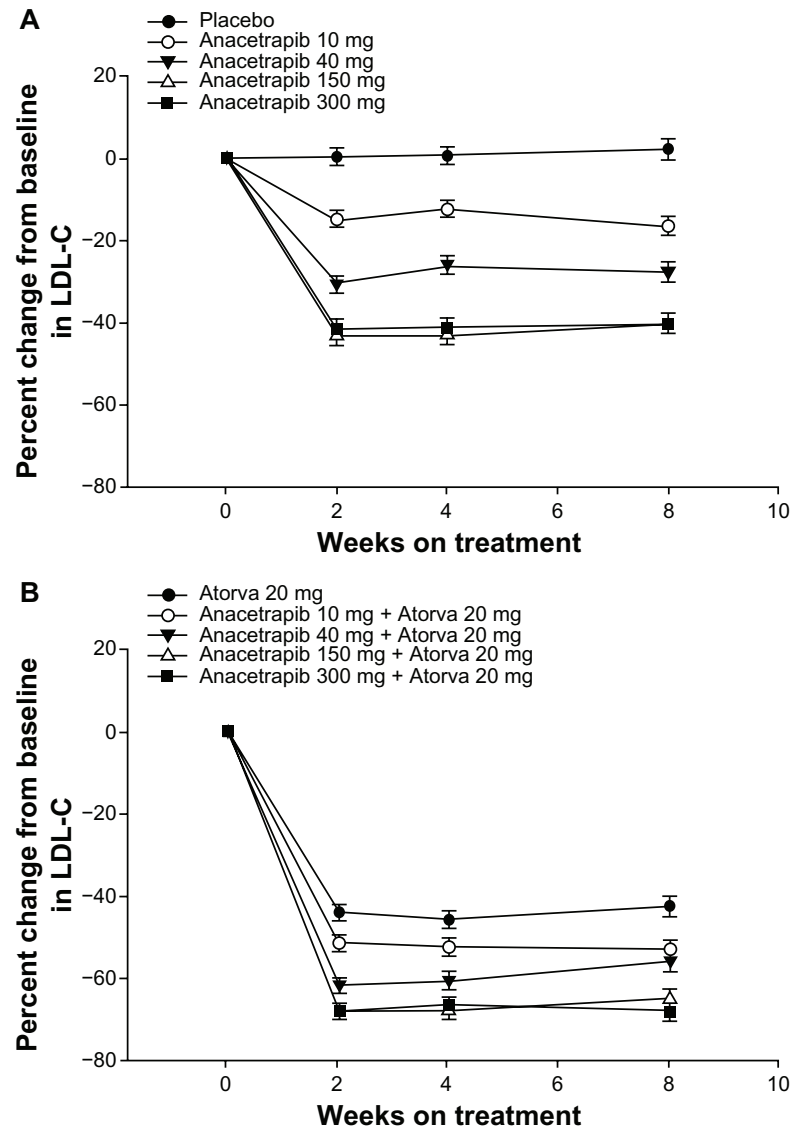

Figure 2 Changes in LDL-C over time. (A) anacetrapib monotherapy versus placebo and (B) anacetrapib + atorvastatin $20 \mathrm{mg}$ versus atorvastatin $20 \mathrm{mg}$. Note: Reprinted from the American Heart Journal. Bloomfield D, Carlson GL, Sapre A, et al. Efficacy and safety of the cholesteryl ester transfer protein inhibitor anacetrapib as monotherapy and coadministered with atorvastatin in dyslipidemic patients. Am Heart J. 2009;157:352-360. Copyright ${ }^{\odot} 2009$, with permission from Elsevier. ${ }^{52}$

Abbreviation: LDL-C, low-density lipoprotein cholesterol.

monotherapy and combination therapy well, as the incidence of adverse effects were similar between the groups. There were no deaths or serious adverse effects. The most common complaint included constipation, diarrhea, dyspepsia, and myalgia. Most notably, there was no effect on either SBP or DBP (Figure 4). Although this study revealed significant positive alterations in the lipid profile with anacetrapib alone or when coadministered with atorvastatin, further studies are required to demonstrate how these surrogate markers will translate into positive $\mathrm{CV}$ outcomes.

Krauss et al examined the effects of anacetrapib on plasma lipids, lipoprotein subfraction concentrations, and lipoprotein composition in 30 healthy individuals following 14 days of therapy. Patients were randomized to receive anacetrapib $150 \mathrm{mg}$ daily, $20 \mathrm{mg}$ daily, or placebo. In patients receiving $150 \mathrm{mg}$ of anacetrapib, LDL-C was reduced by $26 \%$ and HDL-C was increased by $82 \%$. Additionally, there was a $29 \%$ decrease in apo B, a $21 \%$ increase in apo A-I, and a $43 \%$ decrease in lipoprotein (a). Regarding particle 

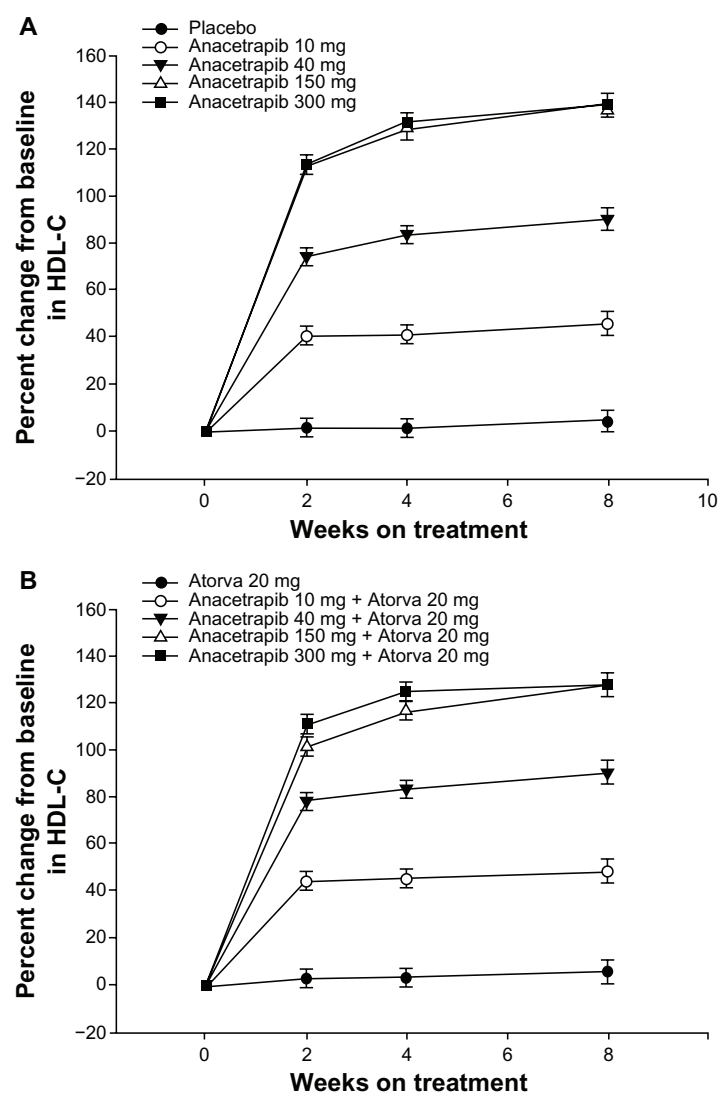

Figure 3 Changes in HDL-C over time. (A) Anacetrapib monotherapy versus placebo and (B) anacetrapib + atorvastatin $20 \mathrm{mg}$ versus atorvastatin $20 \mathrm{mg}$. Note: Reprinted from the American Heart Journal. Bloomfield D, Carlson GL, Sapre A, et al. Efficacy and safety of the cholesteryl ester transfer protein inhibitor anacetrapib as monotherapy and coadministered with atorvastatin in dyslipidemic patients. Am Heart J. 2009;157:352-360. Copyright ${ }^{\odot} 2009$, with permission from Elsevier. ${ }^{52}$ Abbreviation: HDL-C, high-density lipoprotein cholesterol.

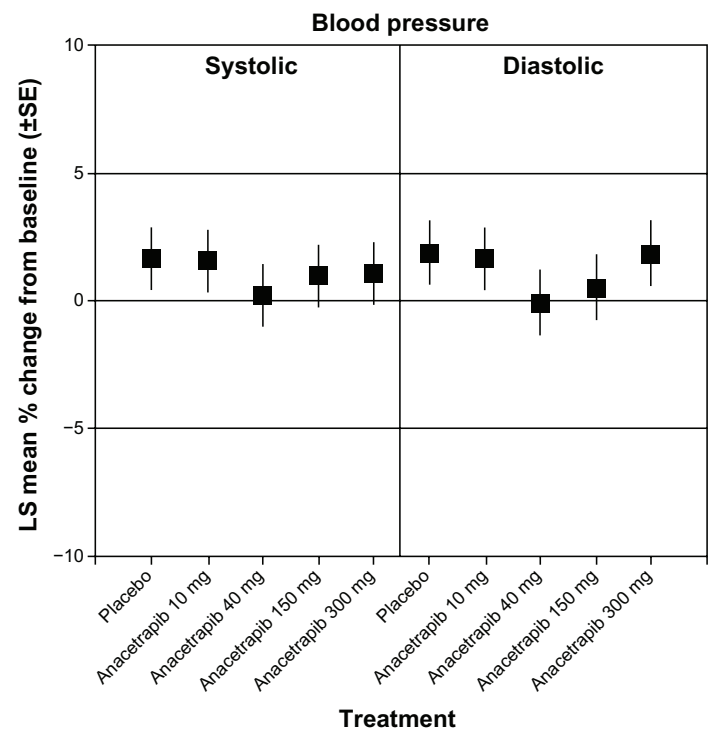

Figure 4 Changes in systolic and diastolic blood pressure at week 8 with anacetrapib monotherapy versus placebo.

Note: Reprinted from the American Heart Journal. Bloomfield D, Carlson GL, Sapre A, et al. Efficacy and safety of the cholesteryl ester transfer protein inhibitor anacetrapib as monotherapy and coadministered with atorvastatin in dyslipidemic patients. Am Heart J. 2009;157:352-360. Copyright ${ }^{\odot} 2009$, with permission from Elsevier. ${ }^{52}$

Abbreviations: LS, least squares; SE, standard error. protein analysis, anacetrapib $150 \mathrm{mg}$ once daily significantly reduced baseline mean particle concentrations of medium VLDL (22\%), small VLDL (31\%), large intermediate density lipoproteins (35\%), medium LDL2a (35\%), LDL2b (39\%), and small LDL3a (28\%) when compared to placebo. In addition, there was a significant increase in particle concentration of very small LDL4b (75\%). Lastly, anacetrapib $150 \mathrm{mg}$ significantly increased large HDL $2 \mathrm{~b}$ by $373 \%$, yet yielded no significant change in smaller HDL $2 \mathrm{a}+3$ particle concentrations. $^{53}$

A model-based approach was used to identify a suitable dose for future trials. ${ }^{54}$ Considering the various variables examined (ie, formulation, diet, and study population), a dose of $100 \mathrm{mg}$ for anacetrapib was selected for future study in Phase III trials that was not previously analyzed in the Bloomfield et al Phase IIb trial. The lipid-altering effects demonstrated in a Phase III trial, which was published during the review of the model-based approach, validated the predictions made..$^{55}$

\section{DEFINE trial}

Unlike the trial by Bloomfield et al, patients in The Determining the Efficacy and Tolerability of CETP Inhibition with Anacetrapib (DEFINE) study were included if they had pre-existing CHD or were at high risk of CHD. ${ }^{55,56}$ This international, double-blind, Phase III, placebo-controlled trial evaluated the lipid level effects, tolerability, and safety profile of anacetrapib. Of the 2757 patients who were initially screened, 1623 patients were randomized to receive anacetrapib $100 \mathrm{mg}$ or placebo daily in combination with statin therapy with or without other antilipemic agents. Each of the groups included patients aged 18 to 80 years who had LDL-C between 50 to $100 \mathrm{mg} / \mathrm{dL}$, HDL-C less than $60 \mathrm{mg} / \mathrm{dL}$, and TG not more than $400 \mathrm{mg} / \mathrm{dL}$. The majority of patients had CHD, while $45.3 \%$ had risk factors for CHD. Only $0.7 \%$ of the patients were not on statin treatment.

The efficacy endpoints in the study were the percent change from baseline within the two treatment groups in LDL-C at 24 weeks and the safety and tolerability profile of anacetrapib during the 76 weeks. As in the Bloomfield et al trial, LDL-C was chosen as a primary endpoint because it is a $\mathrm{CV}$ risk factor that anacetrapib impacts. The change in LDL-C up to week 76 and change in HDL-C, non-HDL-C, apo B, and apo A-I after 24 weeks and 76 weeks of therapy were secondary efficacy endpoints. In addition to the safety endpoints of CV death, nonfatal myocardial infarction, stroke, and hospitalization due to unstable angina, other variables evaluated included blood pressure and electrolyte levels. The DEFINE 
trial analyzed the CV endpoints via a Bayesian approach to exclude the $25 \% \mathrm{CV}$ events that occurred with torcetrapib. After 24 weeks of treatment, DEFINE established both a statistically significant decrease in LDL-C and increase in HDL-C over placebo (Figure 5). These effects extended to week 76. Although secondary efficacy outcomes were also positively affected versus placebo, confirmation of a lack of benefit with C-reactive protein levels was established. Furthermore, the Bayesian analysis confirmed a 94\% probability that anacetrapib would not produce a $25 \%$ increase in $\mathrm{CV}$ adverse outcomes previously detected with torcetrapib (Table 1). Although not all the surrogate markers originally used to test the off-target effects of torcetrapib have been analyzed with anacetrapib, the positive $\mathrm{CV}$ results of the DEFINE trial have assisted in reinvestigating the hypothesis that CETP inhibition is cardioprotective. Ideally, a large- scale, randomized, placebo-controlled trial representing a variety of ethnic groups while evaluating the effects of long-term reduction of LDL-C to very low levels should be conducted in order to offer definitive outcomes as to the safety and efficacy of anacetrapib.

\section{REVEAL trial}

Anacetrapib is currently under investigation in the Randomized Evaluation of the Effects of Anacetrapib through Lipid Modification (REVEAL), a large-scale, randomized, placebocontrolled trial of the clinical effects of anacetrapib among people with established vascular disease. ${ }^{57}$ This double-blind Phase III trial of anacetrapib $100 \mathrm{mg}$ daily will test the hypothesis if anacetrapib will reduce the incidence of major coronary events (ie, coronary death, myocardial infarction, or coronary revascularization procedure) in patients with a
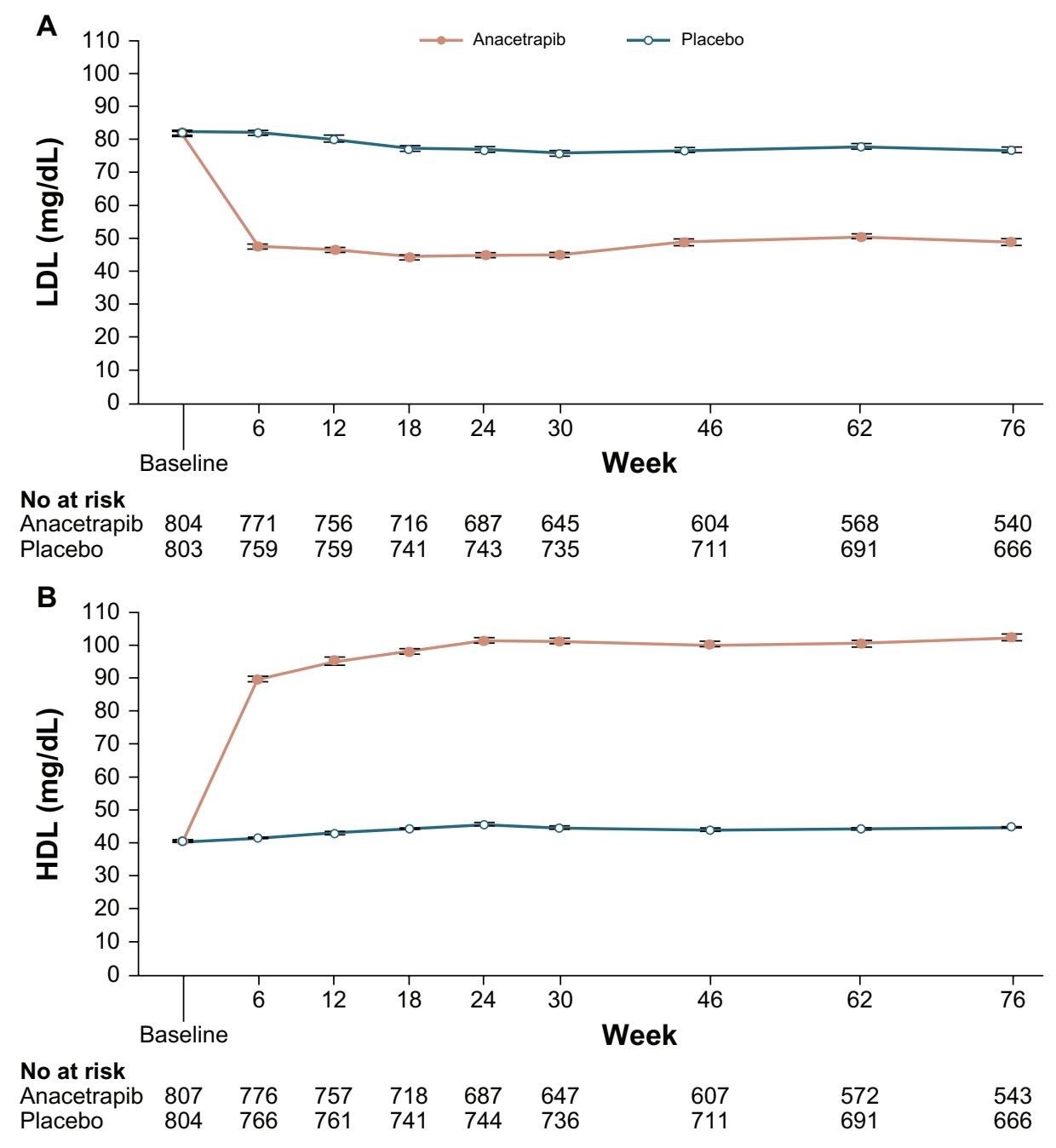

Figure 5 Changes in cholesterol levels during the study period.

Notes: LDL-C and HDL-C levels in the anacetrapib and placebo groups from baseline to week 76. Horizontal bars indicate standard error. To convert the values for LDL-C and HDL-C to mmol/L, multiply by 0.02586 . Reprinted from Cannon CP, Shah S, Dansky HM, et al. Determining the Efficacy and Tolerability Investigators. Safety of anacetrapib in patients with or at high risk for coronary heart disease. N Engl J Med. 2010;363(25):2406-24I5. Copyright ${ }^{\odot} 2010$, Massachusetts Medical Society. Reprinted with permission from Massachusetts Medical Society. ${ }^{56}$

Abbreviations: HDL-C, high-density lipoprotein cholesterol; LDL-C, low-density lipoprotein cholesterol. 
Table I Cardiovascular Events during the Treatment Phase of the Study*

\begin{tabular}{lll}
\hline Event & $\begin{array}{l}\text { Anacetrapib } \\
(\mathbf{N}=\mathbf{8 0 8})\end{array}$ & $\begin{array}{l}\text { Placebo } \\
\mathbf{( N = 8 0 4 )}\end{array}$ \\
\hline & $\begin{array}{l}\text { Number (percent) } \\
16(2.0)\end{array}$ & $2 \mathrm{I}(2.6)$ \\
$\begin{array}{l}\text { Prespecified, adjudicated cardiovascular } \\
\text { safety end point }\end{array}$ & & \\
$\quad$ Death from cardiovascular causes & $4(0.5)$ & $\mathrm{I}(0.1)$ \\
Nonfatal myocardial infarction & $6(0.7)$ & $9(1.1)$ \\
Hospitalization for unstable angina & $\mathrm{I}(0.1)$ & $6(0.7)$ \\
Nonfatal stroke & $5(0.6)$ & $5(0.6)$ \\
Death from any cause & $\mathrm{II}(\mathrm{I} .4)$ & $8(1.0)$ \\
Heart failure & $3(0.4)$ & $4(0.5)$ \\
Revascularization & $8(\mathrm{I} .0)$ & $28(3.5)$ \\
PCI & $6(0.7)$ & $25(3.1)$ \\
CABG & $2(0.2)$ & $3(0.4)$ \\
\hline
\end{tabular}

Notes: $*$ The duration of the treatment phase of the study was 76 weeks. Reprinted from Cannon CP, Shah S, Dansky HM, et al. Determining the Efficacy and Tolerability Investigators. Safety of anacetrapib in patients with or at high risk for coronary heart disease. N Engl J Med. 2010;363(25):2406-24I5. Copyright ${ }^{\odot} 2010$, Massachusetts Medical Society. Reprinted with permission from Massachusetts Medical Society. ${ }^{56}$ Abbreviations: $\mathrm{CABG}$, coronary-artery bypass grafting; $\mathrm{PCl}$, percutaneous coronary intervention.

history of CVD who are taking statin therapy for lowering LDL-C.

The REVEAL trial is currently enrolling patients 50 years or older who meet at least one of the following inclusion criteria: history of myocardial infarction, cerebrovascular atherosclerotic disease, or peripheral arterial disease; or diabetes mellitus with other evidence of symptomatic CHD (ie, treatment or hospitalization for angina or a history of coronary revascularization or acute coronary syndrome). This international multicenter trial is expected to enroll 30,000 patients and is estimated to be completed by $2017 .{ }^{57}$

\section{Alternative agents to raise HDL-C}

There are three agents in the quiver of currently available pharmacological agents to promote raising HDL-C: nicotinic acid (niacin), fibric acid derivatives (fibrates), and statins. Of these agents, niacin has a more pronounced effect on HDLC, raising HDL-C by $15 \%$ to $35 \%$. In addition to its effects on HDL-C, niacin has also been shown to decrease LDL-C by $5 \%$ to $25 \%$ and decrease TGs by $20 \%$ to $50 \%$. $^{2}$ Niacin is believed to increase circulating HDL-C by inhibiting the uptake and catabolism of HDL-apo A within the liver without altering HDL-apo A synthesis. By reducing the catabolic rate, niacin prolongs the half-life of HDL-C, allowing for greater accumulation of cholesterol and larger, more antiatherogenic, HDL-C molecules. ${ }^{58,59}$ Additionally, niacin has also been shown to indirectly increase HDL-C by decreasing the activity of CETP. ${ }^{60}$
Niacin is available in three oral formulations: immediate release, extended release, and sustained release. Traditional immediate-release niacin is dosed two to three times daily, whereas the newer extended-release formulations can be administered once daily. The most common adverse effects associated with niacin, which may affect tolerability and adherence, include diarrhea, nausea, vomiting, increased cough, pruritus, and most notably flushing of the face and upper body. Niacin-induced flushing is believed to result from rapid elevations in serum nicotinic acid. In an effort to prevent these rapid elevations, extended release formulations of niacin have been created. The incidence and severity of flushing may be reduced by administering aspirin 30 minutes prior to niacin. Additionally, the dosage of niacin should be slowly titrated to minimize this unwanted adverse effect. It should be noted that tolerance to the incidence and severity of niacin develops over several weeks following initiation. ${ }^{61,62}$

Despite niacin's ability to substantially increase HDL$\mathrm{C}$, evidence to support the efficacy of niacin to reduce $\mathrm{CV}$ outcomes is inconsistent. A 2010 meta-analysis of niacin alone or in combination with other lipid-lowering agents concluded that the use of niacin significantly reduced major coronary events, stroke, and any CV events. ${ }^{63}$ Several of the studies included in the analysis took place before statin therapy became the standard of care. In contrast to this metaanalysis, the Atherothrombosis Intervention in Metabolic Syndrome with Low HDL Cholesterol/High Triglyceride and Impact on Global Health Outcomes (AIM-HIGH) trial, which evaluated the effects of extended release niacin in addition to intensive statin therapy in the prevention of CV events, was terminated early due to a lack of efficacy. ${ }^{64}$ Results of AIMHIGH have called into question the benefits of increasing HDL-C with niacin in addition to intensive statin therapy.

Although fibrates (fenofibrate, fenofibric acid, gemfibrozil) exert a more profound effect on TGs (20\% to $50 \%$ reduction), these agents have been shown to increase HDL-C levels by $10 \%$ to $35 \%$ and also lower LDL-C by $5 \%$ to $20 \% .^{2}$ The exact mechanism by which fibrates exert their lipid-modifying capabilities has not been fully determined; however, it is understood that these agents activate peroxisome proliferator-activated receptor- $\alpha$ (PPAR- $\alpha$ ). Fibrate-mediated activation of PPAR- $\alpha$ results in both upregulation and downregulation of several genes that partake in lipid metabolism and transport within the liver and adipose tissue. ${ }^{65}$ In addition to the aforementioned atherosclerotic benefits of fibrates, these agents are believed to possess several pleiotropic effects. Fibrates, in particular, have been shown to modulate pro-inflammatory cytokines 
as well as fibrinogen, plasminogen activator inhibitor-1, and C-reactive protein - each linked to atherosclerosis. ${ }^{66}$

Within the US, there are two Food and Drug Administration (FDA) approved fibrates: gemfibrozil and fenofibrate. FDAapproved derivates of fenofibrate have also been developed. These include micronized fenofibrate and fenofibric acid. Owing to its preference among patients and physicians, fenofibrate is administered once daily, whereas gemfibrozil is given twice daily with meals. Although fibrates are generally well tolerated, the most commonly reported side effects are gastrointestinal upset, dyspepsia, abdominal pain, cramping, muscle aches, and rash. A major disadvantage of gemfibrozil is the increased risk of rhabdomyolysis when administered with statins. Glucuronidation, a pathway for renal excretion of lipophilic statins, is significantly inhibited by gemfibrozil but not fenofibrate. ${ }^{67}$ Therefore, fenofibrate is preferred in those who require combined therapy with a statin and fibrate. ${ }^{68-70}$

Similarly to niacin, the effect of raising HDL-C with fibrates has not consistently resulted in positive $\mathrm{CV}$ outcomes. In the Veterans Affairs HDL Intervention Trial study, the use of gemfibrozil to raise HDL-C and lower TG levels in patients with a history of CHD and an LDL-C level of less than $140 \mathrm{mg} / \mathrm{dL}$ decreased the rate of nonfatal myocardial infarction and death from $\mathrm{CHD}$ by $22 \%{ }^{71}$ Further analysis of this study revealed that the concentrations of HDL-C achieved in the gemfibrozil treatment group were strongly correlated with significant reductions in coronary events. ${ }^{72}$ However, there are rather disheartening results from the Action to Control Cardiovascular Risk in Diabetes (ACCORD) lipid study. In this landmark trial, the addition of fenofibrate to open-label simvastatin therapy in highrisk diabetic patients failed to reduce the rate of $\mathrm{CV}$ events. ${ }^{73}$ Disappointing results of the AIM-HIGH and ACCORD studies have clouded the previously clear correlation between raising HDL-C and preventing CV endpoints.

Of the agents noted to have an impact on serum HDL$\mathrm{C}$, statins have been shown to have the smallest effect, raising HDL-C by $5 \%$ to $15 \% .^{2}$ Within the statin realm, rosuvastatin and atorvastatin have the greatest potential for raising HDL-C. ${ }^{74}$ Statins display their effect on cholesterol by competitively inhibiting $\mathrm{HMG}-\mathrm{CoA}$ reductase, the rate limiting step in cholesterol synthesis. Reductions in hepatocyte cholesterol concentrations result in increased expression of LDL receptors, which promote the removal of circulating LDL-C and LDL precursors. In addition, statins may lower LDL-C independent of LDL receptors by inhibiting the synthesis and secretion of apo B lipoproteins and TG-rich lipoproteins from the liver. ${ }^{75}$ Although statins do in fact raise $\mathrm{HDL}-\mathrm{C}$, the $\mathrm{CV}$ benefit seen with this class is believed to be attributed to reductions in LDL-C as well as their aforementioned pleiotropic effects.

There are currently seven available statins approved for use within the US: atorvastatin, fluvastatin, lovastatin, pitavastatin, pravastatin, rosuvastatin, and simvastatin, each of which are administered once daily. Because the majority of cholesterol synthesis occurs at night, it is recommended that agents with shorter half-lives, such as simvastatin, be administered in the evening or at bedtime. Statins are well tolerated in most patients, and their adverse effects can be seen with each agent throughout the class. The most common adverse effects of statins include myalgia, myopathy, gastrointestinal discomfort, and elevations in liver transaminases. One of the more serious side effects seen with statins is rhabdomyolysis. Therefore, it is of utmost importance that patients newly initiated on statins be monitored for myopathy. Once identified, patients experiencing myopathy should have their statin dose decreased or if not possible, the medication should be discontinued. Additionally, concomitant administration of CYP3A4 inhibitors increase the risk of myopathy and rhabdomyolysis; thus, these patients should be monitored more scrupulously. ${ }^{75}$

\section{Conclusion}

The function of CETP is vital to lipid metabolism, and inhibition of this glycoprotein signifies a potential strategy to manage dyslipidemia. Compared to the current FDA-approved agents, anacetrapib increases HDL-C levels above that observed with niacin or fibrates. The safety and tolerability of anacetrapib was confirmed in multiple studies including DEFINE, and the off-target adverse effects previously reported with torcetrapib were not replicated with anacetrapib. While the place in therapy of niacin and fibrates is currently in question, the ongoing outcomes of the REVEAL study will not only test the hypothesis if CETP inhibition lowers residual $\mathrm{CV}$ risk but also provide insight as to which patient subgroups might benefit the most from anacetrapib despite aggressive therapy with statins. Pending the results of the REVEAL trial, anacetrapib may potentially be the first FDA-approved agent in this class to treat patients with dyslipidemia and those with proven atherosclerotic CVD.

\section{Disclosure}

The authors report no conflicts of interest in this work.

\section{References}

1. Heidenreich PA, Trogdon JG, Khavjou OA, et al. Forecasting the future of cardiovascular disease in the United States: a policy statement from the American Heart Association. Circulation. 2011;123(8):933-944. 
2. Expert Panel on Detection, Evaluation, and Treatment of High Blood Cholesterol in Adults. Executive Summary of the Third Report of the National Cholesterol Education Program (NCEP) Expert Panel on Detection, Evaluation, and Treatment of High Blood Cholesterol in Adults (Adult Treatment Panel III). JAMA. 2001;285(19):2486-2497.

3. Smith SC Jr, Allen J, Blair SN, et al. AHA/ACC guidelines for secondary prevention for patients with coronary and other atherosclerotic vascular disease: 2006 update: endorsed by the National Heart, Lung, and Blood Institute. Circulation. 2006;113(19):2363-2372.

4. Heart Protection Study Collaborative Group. MRC/BHF Heart Protection Study of cholesterol lowering with simvastatin in 20,536 high-risk individuals: a randomized placebo-controlled trial. Lancet. 2002;360(9326):7-22.

5. Ridker PM, Danielson E, Fonseca FA, et al. Rosuvastatin to prevent vascular events in men and women with elevated C-reactive protein. N Engl J Med. 2008;359(21):2195-2207.

6. Scandinavian Simvastatin Survival Study Group. Baseline serum cholesterol and treatment effect in the Scandinavian Simvastatin Survival Study (4S). Lancet. 1995;345(8960):1274-1275.

7. Davignon J. Beneficial cardiovascular pleiotropic effects of statins. Circulation. 2004;109(23 Suppl 1):III39-III43.

8. Barter P, Gotto AM, LaRosa JC; for Treating to New Targets Investigators. HDL cholesterol, very low levels of LDL cholesterol, and cardiovascular events. N Engl J Med. 2007;357(13):1301-1310.

9. Gordon T, Castelli WP, Hjortland MC, Kannel WB, Dawber TR. High density lipoprotein as a protective factor against coronary heart disease. The Framingham Study. Am J Med. 1977;62(5):707-714.

10. Assmann G, Schulte H, von Eckardstein A, Huang Y. High-density lipoprotein cholesterol as a predictor of coronary heart disease risk. The PROCAM experience and pathophysiological implications for reverse cholesterol transport. Atherosclerosis. 1996;124 Suppl:S11-S20.

11. Goldbourt U, Medalie JH. High density lipoprotein cholesterol and incidence of coronary heart disease - the Israeli Ischemic Heart Disease Study. Am J Epidemiol. 1979;109(3):296-308.

12. Miller NE, Thelle DS, Forde OH, Mjos OD. The Tromsø heart-study. High-density lipoprotein and coronary heart-disease: a prospective case-control study. Lancet. 1977;1(8019):965-968.

13. Gordon DJ, Probstfield JL, Garrison RJ, et al. High-density lipoprotein cholesterol and cardiovascular disease. Four prospective American studies. Circulation. 1989;79(1):8-15.

14. Duffy D, Rader DJ. Emerging therapies targeting high-density lipoprotein metabolism and reverse cholesterol transport. Circulation. 2006;113:1140-1150.

15. Shah PK. Emerging HDL-based therapies for atherothrombotic vascular disease. Curr Treat Options Cardiovasc Med. 2007;9(1):60-70.

16. Clark RW, Sutfin TA, Ruggeri RB, et al. Raising high-density lipoprotein in humans through inhibition of cholesteryl ester transfer protein: an initial multidose study of torcetrapib. Arterioscler Thromb Vasc Biol. 2004;24(3):490-497.

17. Brousseau ME, Schaefer EJ, Wolfe ML, et al. Effects of an inhibitor of cholesteryl ester transfer protein on HDL cholesterol. NEngl J Med. 2004;350:1505-1515.

18. Davidson MH, McKenney JM, Shear CL, Revkin JH. Efficacy and safety of torcetrapib, a novel cholesteryl ester transfer protein inhibitor, in individuals with below-average high-density lipoprotein cholesterol levels. J Am Coll Cardiol. 2006;48:1774-1781.

19. McKenney JM, Davidson MH, Shear CL, Revkin JH. Efficacy and safety of torcetrapib, a novel cholesteryl ester transfer protein inhibitor, in individuals with below-average high-density lipoprotein cholesterol levels on a background of atorvastatin. J Am Coll Cardiol. 2006;48:1782-1790.

20. Barter PJ, Caulfield M, Eriksson M, et al. Effects of torcetrapib in patients at high risk for coronary events. $N$ Engl J Med. 2007;357:2109-2122.

21. Nissen SE, Tardif JC, Nicholls SJ, et al. Effect of torcetrapib on the progression of coronary atherosclerosis. N Engl J Med. 2007;356:1304-1316.

22. Kastelein J, van Leuven SI, Burgess L, et al. Effect of torcetrapib on carotid atherosclerosis in familial hypercholesterolemia. NEngl J Med. 2007;356:1620-1630.
23. Bots ML, Visseren FL, Evans GW, et al. Torcetrapib and carotid intimamedia thickness in mixed dyslipidaemia (RADIANCE 2 study): a randomised, double-blind trial. Lancet. 2007;370(9582):153-160.

24. Kuivenhoven JA, de Grooth GJ, Kawamura H, et al. Effectiveness of inhibition of cholesteryl ester transfer protein by JTT-705 in combination with pravastatin in type II dyslipidemia. Am J Cardiol. 2005;95:1085-1088.

25. Krishna R, Anderson MS, Bergman AJ, et al. Effect of the cholesteryl ester transfer protein inhibitor, anacetrapib, on lipoproteins in patients with dyslipidaemia and on 24-h ambulatory blood pressure in healthy individuals: two double-blind, randomized placebo-controlled phase I studies. Lancet. 2007;370(9603):1907-1914.

26. Forrest MJ, Bloomfield D, Briscoe RJ, et al. Torcetrapib-induced blood pressure elevation is independent of CETP inhibition and is accompanied by increased circulating levels of aldosterone. Br J Pharmacol. 2008;154:1465-1473.

27. Vergeer M, Stroes ES. The pharmacology and off-target effects of some cholesterol ester transfer protein inhibitors. Am J Cardiol. 2009; 104 Suppl:32E-38E.

28. Hoffmann-La Roche. A randomized, double-blind, placebo-controlled study assessing the effect of ro4607381 on cardiovascular mortality and morbidity in clinically stable patients with a recent acute coronary syndrome. In: ClinicalTrials.gov [Internet]. Bethesda, MD: National Library of Medicine; 2000. [cited May 28, 2012]. Available from: http://clinicaltrials.gov/show/NCT00658515. NLM Identifier: NCT00658515.

29. Ranalletta M, Bierilo KK, Chen Y, et al. Biochemical characterization of cholesteryl ester transfer protein inhibitors. J Lipid Res. 2010;51(9):2739-2752.

30. Goedeke L, Fernández-Hernando C. Regulation of cholesterol homeostasis. Cell Mol Life Sci. 2012;69(6):915-930.

31. Barter PJ, Kastelein JJ. Targeting cholesteryl ester transfer protein for the prevention and management of cardiovascular disease. J Am Coll Cardiol. 2006;47(3):492-499.

32. Chapman MJ. Therapeutic elevation of HDL-cholesterol to prevent atherosclerosis and coronary heart disease. Pharmacol Ther. 2006;111(3):893-908.

33. Toth PP. Reverse cholesterol transport: high-density lipoprotein's magnificent mile. Curr Atheroscler Rep. 2003;5:386-393.

34. Oliveira HC, de Faria EC. Cholesteryl ester transfer protein: the controversial relation to atherosclerosis and emerging new biological roles. IUBMB Life. 2011;63(4):248-257.

35. Shah PK. Inhibition of CETP as a novel therapeutic strategy for reducing the risk of atherosclerotic disease. Eur Heart J. 2007;28(1):5-12.

36. Chait A, Brazg RL, Tribble DL, Krauss RM. Susceptibility of small, dense, low-density lipoproteins to oxidative modification in subjects with the atherogenic lipoprotein phenotype, pattern B. Am J Med. 1993;94(4):350-356.

37. Krauss RM. Heterogeneity of plasma low-density lipoproteins and atherosclerosis risk. Curr Opin Lipidol. 1994;5(5):339-349.

38. Hirano K, Yamashita S, Kuga Y, et al. Atherosclerotic disease in marked hyperalphalipoproteinemia. Combined reduction of cholesteryl ester transfer protein and hepatic triglyceride lipase. Arterioscler Thromb Vasc Biol. 1995;15:1849-1856.

39. Zhong S, Sharp DS, Grove JS, et al. Increased coronary heart disease in Japanese-American men with mutation in the cholesteryl ester transfer protein gene despite increased HDL levels. J Clin Invest. 1996;97(12):2917-2923.

40. Ridker PM, Paré G, Parker AN, Zee RY, Miletich JP, Chasman DI. Polymorphism in the CETP gene region, HDL cholesterol, and risk of future myocardial infarction: Genomewide analysis among 18245 initially healthy women from the Women's Genome Health Study. Circ Cardiovasc Genet. 2009;2(1):26-33.

41. Curb JD, Abbott RD, Rodriguez BL, et al. Prospective study of HDL-C and cholesteryl ester transfer protein gene mutations and the risk of coronary heart disease in the elderly. J Lipid Res. 2004;45(5):948-953.

42. Tan EY, Hartmann G, Chen Q, et al. Pharmacokinetics, metabolism, and excretion of anacetrapib, a novel inhibitor of the cholesteryl ester transfer protein, in rats and rhesus monkeys. Drug Metab Dispos. 2010;38:459-473. 
43. Krishna R, Garg A, Panebianco D, et al. Single-dose pharmacokinetics and pharmacodynamics of anacetrapib, a potent cholesteryl ester transfer protein (CETP) inhibitor, in healthy subjects. Br J Clin Pharmacol. 2009;68:535-545.

44. Krishna R, Bergman AJ, Jin B, et al. Multiple-dose pharmacodynamics and pharmacokinetics of anacetrapib, a potent cholesteryl ester transfer protein (CETP) inhibitor, in healthy subjects. Clin Pharmacol Ther. 2008;84:679-683.

45. Kumar S, Tan EY, Hartmann G, et al. Metabolism and excretion of anacetrapib, a novel inhibitor of the cholesteryl ester transfer protein, in humans. Drug Metab Dispos. 2010;38:474-483.

46. Dansky HM, Bloomfield D, Gibbons P, et al. Efficacy and safety after cessation of treatment with the cholesteryl ester transfer protein inhibitor anacetrapib (MK-0859) in patients with primary hypercholesterolemia or mixed hyperlipidemia. Am Heart J. 2011;162(4):708-716.

47. Krishna R, Bergman AJ, Jin B, et al. Assessment of the CYP3A-mediated drug interaction potential of anacetrapib, a potent cholesteryl ester transfer protein (CETP) inhibitor, in healthy volunteers. J Clin Pharmacol. 2009;49(1):80-87.

48. Krishna R, Garg A, Jin B, et al. Assessment of a pharmacokinetic and pharmacodynamic interaction between simvastatin and anacetrapib, a potent cholesteryl ester transfer protein(CETP) inhibitor, in healthy subjects. Br J Clin Pharmacol. 2009;67:520-526.

49. Krishna R, Stypinski D, Ali M, et al. Lack of meaningful effect of anacetrapib on the pharmacokinetics and pharmacodynamics of warfarin in healthy subjects. Br J Clin Pharmacol. 2012;74(1):116-124.

50. Krishna R, Stypinski D, Ali M, et al. Lack of an effect of anacetrapib on the pharmacokinetics of digoxin in healthy subjects. Biopharm Drug Dispos. 2011;32(9):525-529.

51. Clark RW, Ruggeri RB, Cunningham D, Bamberger MJ. Description of the torcetrapib series of cholesteryl ester transfer protein inhibitors, including mechanism of action. J Lipid Res. 2006;47(3):537-552.

52. Bloomfield D, Carlson GL, SapreA, et al. Efficacy and safety of the cholesteryl ester transfer protein inhibitor anacetrapib as monotherapy and coadministered with atorvastatin in dyslipidemic patients. Am Heart J. 2009;157:352-360.

53. Krauss RM, Wojnooski K, Orr J, et al. Changes in lipoprotein subfraction concentration and composition in healthy individuals treated with the CETP inhibitor anacetrapib. J Lipid Res. 2012;53(3):540-547.

54. Krishna R, Bergman AJ, Green M, Dockendorf MF, Wagner JA, Dykstra K. Model-based development of anacetrapib, a novel cholesteryl ester transfer protein inhibitor. AAPS J. 2011;13(2):179-190.

55. Cannon CP, Dansky HM, Davidson M, et al. Design of the DEFINE trial: determining the efficacy and tolerability of CETP inhibition with anacetrapib. Am Heart J. 2009;158(4):513-519.

56. Cannon CP, Shah S, Dansky HM, et al. Determining the Efficacy and Tolerability Investigators. Safety of anacetrapib in patients with or at high risk for coronary heart disease. $N$ Engl J Med. 2010;363(25):2406-2415.

57. Landray M, Bowman L. REVEAL: Randomized EValuation of the Effects of Anacetrapib through Lipid-modification. A large-scale, randomized placebo-controlled trial of the clinical effects of anacetrapib among people with established vascular disease. In: ClinicalTrials. gov [Internet]. Bethesda, MD: National Library of Medicine; 2000 [cited May 28, 2012]. Available from: http://clinicaltrials.gov/show/ NCT01252953. NLM Identifier: NCT01252953.

58. Creider JC, Hegele RA, Joy TR. Niacin: another look at an underutilized lipid-lowering medication. Nat Rev Endocrinol. February 21, 2012. [Epub ahead of print.]

Vascular Health and Risk Management

\section{Publish your work in this journal}

Vascular Health and Risk Management is an international, peerreviewed journal of therapeutics and risk management, focusing on concise rapid reporting of clinical studies on the processes involved in the maintenance of vascular health; the monitoring, prevention and treatment of vascular disease and its sequelae; and the involvement of
59. Zhang LH, Kamanna VS, Ganji SH, Xiong XM, Kashyap ML. Niacin increases HDL biogenesis by enhancing DR4-dependent transcription of ABCA1 and lipidation of apolipoprotein A-I in HepG2 cells. J Lipid Res. 2012;53(5):941-950.

60. van der Hoorn JW, de Haan W, Berbée JF, et al. Niacin increases HDL by reducing hepatic expression and plasma levels of cholesteryl ester transfer protein in APOE*3Leiden. CETP mice. Arterioscler Thromb Vasc Biol. 2008;28(11):2016-2022.

61. Abbott Laboratories. NIASPAN [package insert]. North Chicago, IL: Abbott Laboratories; 2010.

62. Gille A, Bodor ET, Ahmed K, Offermanns S. Nicotinic acid: pharmacological effects and mechanisms of action. Annu Rev Pharmacol Toxicol. 2008;48:79-106.

63. Bruckert E, Labreuche J, Amarenco P. Meta-analysis of the effect of nicotinic acid alone or in combination on cardiovascular events and atherosclerosis. Atherosclerosis. 2010;210(2):353-361.

64. Boden WE, Probstfield JL, Anderson T, et al; for AIM-HIGH Investigators. Niacin in patients with low HDL cholesterol levels receiving intensive statin therapy. N Engl J Med. 2011;365(24):2255-2267.

65. Chapman MJ, Redfern JS, McGovern ME, Giral P. Niacin and fibrates in atherogenic dyslipidemia: pharmacotherapy to reduce cardiovascular risk. Pharmacol Ther. 2010;126(3):314-345.

66. McKeage K, Keating GM. Fenofibrate: a review of its use in dyslipidaemia. Drugs. 2011;71(14):1917-1946.

67. Ballantyne CM, Davidson MH. Possible differences between fibrates in pharmacokinetic interactions with statins. Arch Intern Med. 2003; 163(19):2394-2395.

68. Rosenson RS. Current overview of statin-induced myopathy. Am JMed. 2004;116(6):408-416

69. Goldberg AC, Bays HE, Ballantyne CM, et al. Efficacy and safety of ABT-335 (fenofibric acid) in combination with atorvastatin in patients with mixed dyslipidemia. Am J Cardiol. 2009;103(4):515-522.

70. Khoury N, Goldberg AC. The use of fibric acid derivatives in cardiovascular prevention. Curr Treat Options Cardiovasc Med. 2011;13(4): 335-342.

71. Rubins HB, Robins SJ, Collins D, et al. Gemfibrozil for the secondary prevention of coronary heart disease in men with low levels of high-density lipoprotein cholesterol. Veterans Affairs High-Density Lipoprotein Cholesterol Intervention Trial Study Group. NEngl J Med. 1999;341(6):410-418.

72. Robins SJ, Collins D, Wittes JT, et al; VA-HIT Study Group. Veterans Affairs High-Density Lipoprotein Intervention Trial. Relation of gemfibrozil treatment and lipid levels with major coronary events: VA-HIT: a randomized controlled trial. JAMA. 2001;285(12):1585-1591.

73. Ginsberg HN, Elam MB, Lovato LC, et al; for ACCORD Study Group. Effects of combination lipid therapy in type 2 diabetes mellitus. $N$ Engl J Med. 2010;362(17):1563-1574.

74. Santee J, Lindsey C, Pace H. Relative efficacy of antilipemic agents in non-high-density lipoprotein cholesterol reduction. J Pharm Pract. May 2, 2012. [Epub ahead of print.]

75. Maron DJ, Fazio S, Linton MF. Current perspectives on statins. Circulation. 2000;101(2):207-213.

\section{Dovepress}

metabolic disorders, particularly diabetes. This journal is indexed on PubMed Central and MedLine. The manuscript management system is completely online and includes a very quick and fair peer-review system, which is all easy to use. Visit http://www.dovepress.com/ testimonials.php to read real quotes from published authors. 